\title{
Poliorquidismo: presentación de un caso y revisión de la bibliografía
}

\author{
Garza-Montúfar ME, ${ }^{1}$ Olvera-Posada D²
}

\section{Resumen}

El poliorquidismo es una alteración poco frecuente. Hasta la fecha se han reportado menos de 200 casos en la bibliografía de todo el mundo.

CASO CLÍNICO: paciente de 16 años de edad, que acudió a consulta por tumoración en hemiescroto derecho, no dolorosa. En la exploración física se palparon dos estructuras ovoides y dos tubulares que emergían de cada una, sugerentes de conductos deferentes; no se palpó varicocele. Ante la sospecha de poliorquidia, se realizó un ultrasonido escrotal. El estudio de imagen evidenció dos imágenes: una de $3.7 \times 2.7 \times 2.5 \mathrm{~cm}$ y la otra de $3.7 \times 3 \times 2.8 \mathrm{~cm}$, además de microlitiasis. Debido a que el paciente se encontraba asintomático y dispuesto a llevar un adecuado seguimiento, se decidió vigilancia periódica, mediante visitas médicas y ultrasonido seriados.

CONCLUSIÓN: el diagnóstico de poliorquidia se establece mediante la obtención de biopsias o estudios de imagen (ultrasonido y resonancia). El tratamiento depende de la localización, funcionalidad y clasificación del testículo supernumerario; se recomienda dejar el testículo in situ solo cuando tiene localización intraescrotal y es funcional.

PALABRAS CLAVE: poliorquidismo, testículo supernumerario, poliorquia, masa paratesticular, poliorquidia.

Rev Mex Urol. 2017 March;77(2):143-150.

\section{Polyorchidism: a case presentation and review of the literature}

\section{Garza-Montúfar ME, ${ }^{1}$ Olvera-Posada $\mathrm{D}^{2}$}

\section{Abstract}

BACKGROUND: polyorchidism is a rare alteration, with fewer than 200 cases reported in the literature.

CLINICAL CASE: a 16-year-old adolescent sought medical attention for nonpainful tumor of the right hemiscrotum. Upon physical examination, two ovoid structures were palpated, from which emerged tubular structures suggestive of vasa deferentia. Varicocele was not palpated. Suspecting polyorchidism, a scrotal ultrasound study was carried out that identified two images: one measuring $3.7 \times 2.7 \times 2.5$ $\mathrm{cm}$ and the other $3.7 \times 3 \times 2.8 \mathrm{~cm}$, in addition to microlithiasis. Be-
${ }^{1}$ Departamento de Urología, Hospital General de Zona 33, Monterrey, Nuevo León, México.

${ }^{2}$ Tec Salud, Instituto de Cirugía, San Pedro Garza García, Nuevo León, México.

Recibido: octubre 2016

Aceptado: febrero 2017

Correspondencia

Dra. María Esther Garza Montúfar

egmontufar@hotmail.com

Este artículo debe citarse como

Garza-Montúfar ME, Olvera-Posada D. Poliorquidismo: presentación de un caso y revisión de la bibliografía. Rev Mex Urol. 2017 mar;77(2):143-150. 
cause the patient was asymptomatic and willing to carry out adequate follow-up, periodic surveillance was decided upon, through medical visits and serial ultrasound imaging.

DISCUSSION: polyorchidism diagnosis is made through biopsies and imaging studies (ultrasound and magnetic resonance). Treatment depends on the location, functionality, and classification of the supernumerary testis. It is recommended to leave the testis in situ only when there is intrascrotal location and functionality.

KEY WORDS: polyorchidism, supernumerary testis, paratesticular mass

\footnotetext{
${ }^{1}$ Departamento de Urología, Hospital General de Zona 33, Monterrey, Nuevo León, México.

${ }^{2}$ Tec Salud, Instituto de Cirugía, San Pedro Garza García, Nuevo León, México.
}

Correspondence

Dra. María Esther Garza Montúfar egmontufar@hotmail.com

\section{ANTECEDENTES}

El poliorquidismo, también conocido como poliorquia, poliorquidia o testículo supernumerario, se refiere a la presencia de más de 2 testículos localizados de forma intraescrotal (66 a $75 \%$ ) o extraescrotal (región inguinal y con menor frecuencia en la cavidad abdominal). ${ }^{1,2} \mathrm{Es}$ una anomalía congénita excepcional, pues hasta la fecha existen menos de 200 casos publicados en la bibliografía mundial.

Esta alteración fue inicialmente descrita por Balsius en 1670; Ahlfeld confirmó este hallazgo durante una autopsia en 1880 y Lane reportó el primer caso de testículo supernumerario en 1895, corroborado histológicamente en una intervención quirúrgica. ${ }^{3-5}$ Barrios-Torres describió el primer caso de poliorquidismo en México en 1989; ${ }^{6}$ después de éste, solo se han reportado dos casos más en la bibliografía médica mexicana. ${ }^{3,7}$

El objetivo de este estudio es reportar un caso de poliorquidismo y exponer sus diferentes clasificaciones, con la finalidad de considerar la alteración en el diagnóstico diferencial de lesiones extratesticulares.

\section{CASO CLÍNICO}

Paciente de 16 años de edad, que acudió a consulta por tumoración en hemiescroto derecho, no dolorosa. Al interrogatorio indirecto, los familiares comentaron que la tumoración se manifestó desde el nacimiento, donde el personal médico sugirió el diagnóstico de "lipoma del cordón espermático". A la exploración física se palpó el testículo izquierdo y las estructuras del cordón espermático sin alteraciones. En el hemiescroto derecho se palparon dos estructuras ovoides, de consistencia y tamaño similar a la del testículo izquierdo, además de dos estructuras tubulares que emergían de cada una, sugerentes de conductos deferentes; no se palpó varicocele (Figura 1). Ante la sospecha de poliorquidia, se realizó el ultrasonido escrotal. El estudio de imagen no mostró anomalías en el área inguinal. En el hemiescroto izquierdo se observó una imagen compatible con testículo de ecotextura normal, de $4 \times 3 \times 2.5 \mathrm{~cm}$. En el hemiescroto derecho se observaron dos imágenes (una de $3.7 \times 2.7 \times 2.5 \mathrm{~cm}$ y la otra de $3.7 \times 3 \times 2.8$ $\mathrm{cm})$ con ecotextura similar a la encontrada en lado izquierdo, además de microlitiasis (Figura 2). Debido a que el paciente se en- 


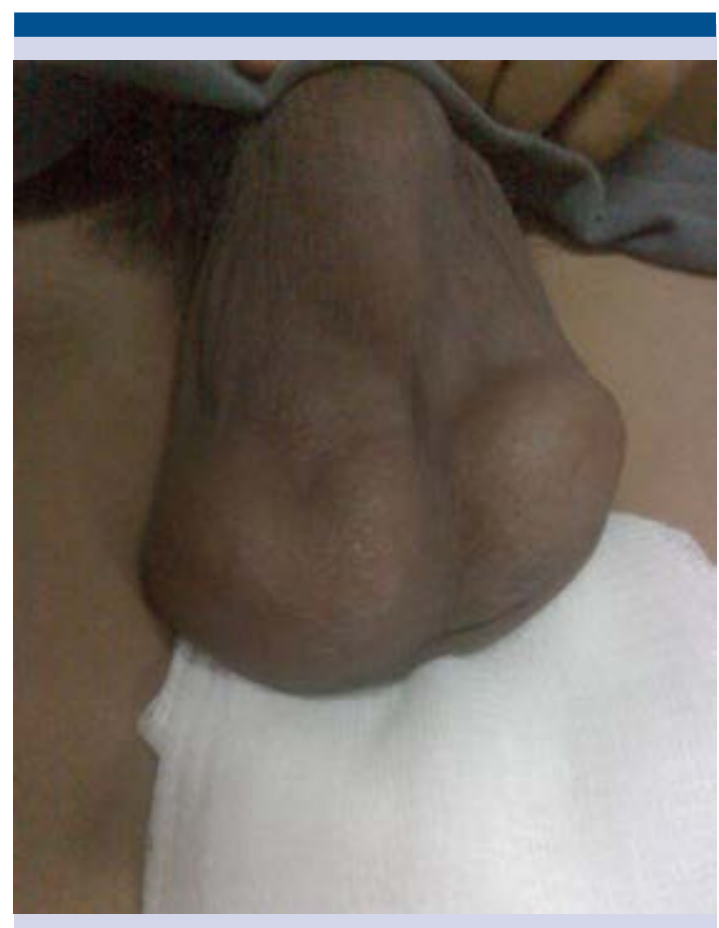

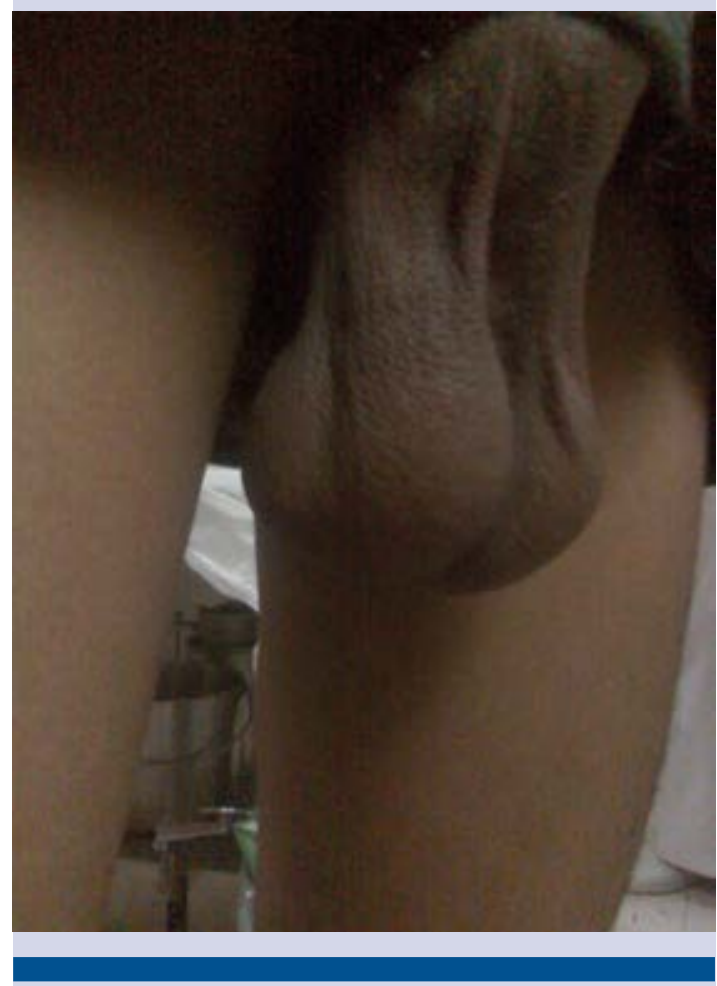

Figura 1. Hemiescroto derecho con dos estructuras ovoides.

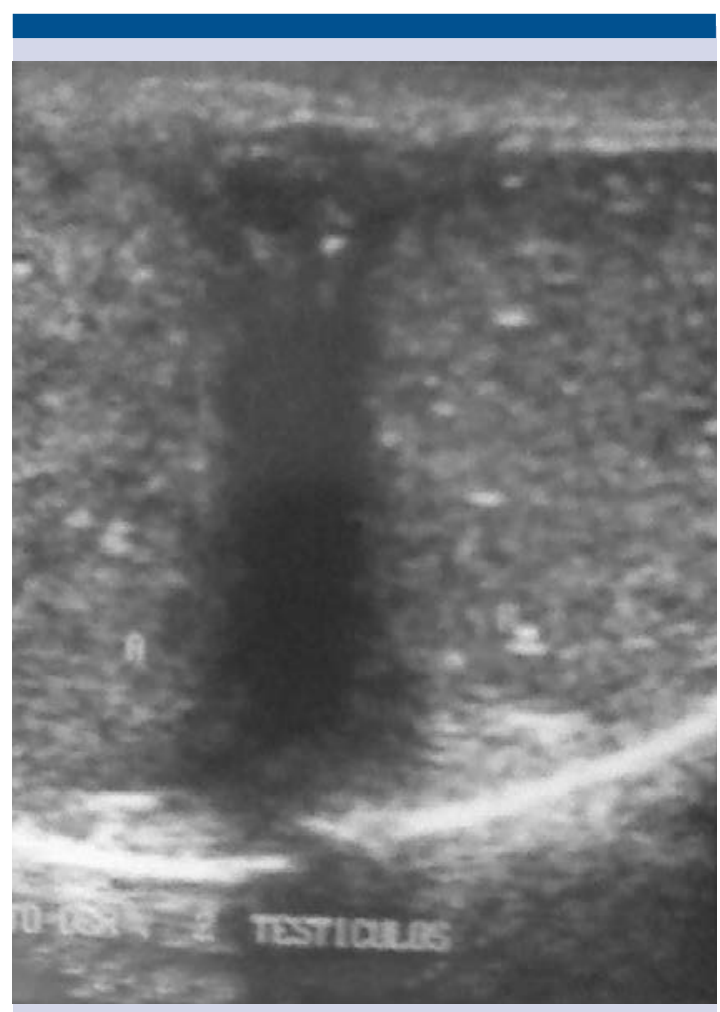

Figura 2. Ultrasonido que muestra una estructura de $3.7 \times 2.7 \times 2.5 \mathrm{~cm}$ y otra de $3.7 \times 3 \times 2.8 \mathrm{~cm}$, además de microlitiasis.

contraba asintomático y dispuesto a llevar un adecuado seguimiento, se decidió vigilancia del testículo supernumerario de manera periódica, mediante visitas médicas y ultrasonido seriados. Hasta el momento (6 años después de establecer el diagnóstico) no ha mostrado ninguna complicación aparente.

\section{DISCUSIÓN}

El poliorquidismo es una alteración poco frecuente, cuya información se ha obtenido sólo de reporte de casos. Un metanálisis publicado por Bergholz $(2009)^{5}$ señala que hasta el 2008 se encontraban reportados 191 casos de poliorquidismo en todo el mundo, de los cuales 140 se confirmaron histológicamente. 
La edad al momento de establecer el diagnóstico varía desde los primeros meses de vida hasta 30 los años (media de 17 años). El sitio más frecuente de poliorquidia es el lado izquierdo, reportado en $65-75 \%$ de los casos. ${ }^{5,8}$ En cuanto al número de testículos respecta, casi siempre se desarrollan tres (dos de ellos en un solo lado), ${ }^{1-4,8-18}$ pocas veces $4(4.3 \%)^{19-22}$ y excepcionalmente 5 testículos, ${ }^{2}$ incluso se han reportado pacientes sin testículo contralateral. ${ }^{23,24}$ Los testículos supernumerarios pueden aparecer de diversas maneras en relación con la presencia o ausencia del epidídimo y conducto deferente, suelen carecer totalmente de estas estructuras, las comparten con el testículo ipsilateral o tienen su propio sistema excretor. La manifestación más frecuente se asocia con testículo supernumerario que comparte el epidídimo y conducto deferente (27\% de los casos). ${ }^{5}$ En el paciente de este estudio se observó un testículo supernumerario con su propio epidídimo y conducto deferente.

La mayoría de los casos se diagnostican de manera incidental, mediante la palpación de una masa en la región inguinal o escrotal, con signos de dolor y hernia inguinal; o al momento de efectuar una intervención quirúrgica por hernia, hidrocele, orquidopexia, etc. En algunos casos puede relacionarse con criptorquidia $(40 \%)$, hernia $(30 \%)$, torsión $(15 \%)$, varicocele o hidrocele $(9 \%)^{2,13}$

\section{Malignidad}

Aún se discuten los criterios de riesgo de malignidad en pacientes con testículo supernumerario. La incidencia de cáncer en sujetos con testículo supernumerario es de $5.7 \%$, principalmente aquellos de localización extraescrotal. Esta incidencia es considerablemente mayor a la estimada en la población general $(0.004 \%)$ y a la de pacientes con testículos no descendidos $(0.045 \%) .{ }^{5}$ La diferencia en la incidencia de ma- lignidad puede deberse, sobre todo, a la posición del testículo supernumerario, que a su misma manifestación; sin embargo, la baja incidencia de esta anomalía congénita impide establecer una conclusión específica.

\section{Diagnóstico}

El diagnóstico clínico, basado solo en exploración física, no es confiable; la manifestación es variable y la sospecha de testículo supernumerario debe considerarse al valorar una masa escrotal o inguinal. ${ }^{17}$ Existen alteraciones escrotales extratesticulares que deben descartarse, como los pseudotumores fibroides. ${ }^{25}$

En reportes iniciales, para establecer el diagnóstico se consideraba sólo el estudio histológico del tejido testicular, ya sea en biopsia incisional o excisional; actualmente se establece sin necesidad de intervención quirúrgica, mediante ultrasonido Doppler, que evidencia el flujo en la estructura sugerente de testículo supernumerario, parénquima testicular y epidídimo, incluso es útil para descartar alguna tumoración en el mismo sitio. En algunos casos se ha realizado resonancia magnética, que brinda mayor información de la alteración, donde el testículo supernumerario tiene las mismas características que uno normal en fases T1 y T2;16 además de identificar la coexistencia de vesículas seminales. En pacientes con sospecha de torsión testicular, también es útil realizar gamagrama. ${ }^{2}$

Cuando el diagnóstico se establece durante alguna intervención quirúrgica, suele observarse tejido testicular en el análisis histológico (tejido resecado en forma incisional o excisional). En otros pacientes se ha considerado la determinación de hormonas como parte del protocolo de estudio; sin embargo, no es necesaria. El diagnóstico diferencial se establece con: quiste de epidídimo o cordón, hidrocele, epidídimo aberrante, tumor paratesticular; cistadenoma, 
lipoma del cordón, calcificaciones escrotales, granulomas, entre otros. ${ }^{2,16}$ En algunas ocasiones los testículos supernumerarios muestran estructuras histológicas normales, pero su espermatogénesis es anormal.

\section{Origen}

Entre las hipótesis relacionadas con el posible origen de testículos supernumerarios se incluyen: división longitudinal o transversal de la cresta genital (secundario a bandas peritoneales), ${ }^{1}$ duplicación de la cresta genital y degeneración incompleta de los componentes mesonéfricos. ${ }^{8,9}$ Esta segmentación ocurre antes de la semana 8 de gestación y según el momento en que se desarrolle, el testículo supernumerario contará con epidídimo y conducto deferente propios o compartidos con el testículo ipsilateral, incluso puede carecer de ambas estructuras anatómicas. ${ }^{2,19}$

\section{Clasificación}

Diversos autores han descrito las clasificaciones con base en aspectos como: origen embriológico, situación anatómica y estado funcional del testículo supernumerario.

Clasificación de Leung $(1988)^{26}$ de acuerdo con su origen embriológico

I) El testículo supernumerario carece de epidídimo y conducto deferente.

II) El testículo supernumerario drena en el epidídimo del testículo ipsilateral y comparten el conducto deferente.

III) El testículo supernumerario tiene su propio epidídimo y comparte el conducto deferente con el testículo ipsilateral.

IV) Duplicación completa del epidídimo y conducto deferente.
Clasificación de Serrano-Brambilla (1996)³ según el desarrollo embriológico (Figura 3)

I) Después de la división temprana de la cresta genital, el testículo supernumerario no se une con los conductos excretores mesonéfricos, por lo que no tiene epidídimo ni conducto deferente.

II) Cuando la división ocurre en una etapa intermedia y el testículo supernumerario se une a los conductos excretores mesonéfricos proximales, tiene propio epidídimo y el conducto deferente en común con el testículo ipsilateral.

III) Cuando la división de la cresta genital ocurre de manera tardía, el testículo supernumerario y el ipsilateral comparten el mismo epidídimo y conducto deferente.

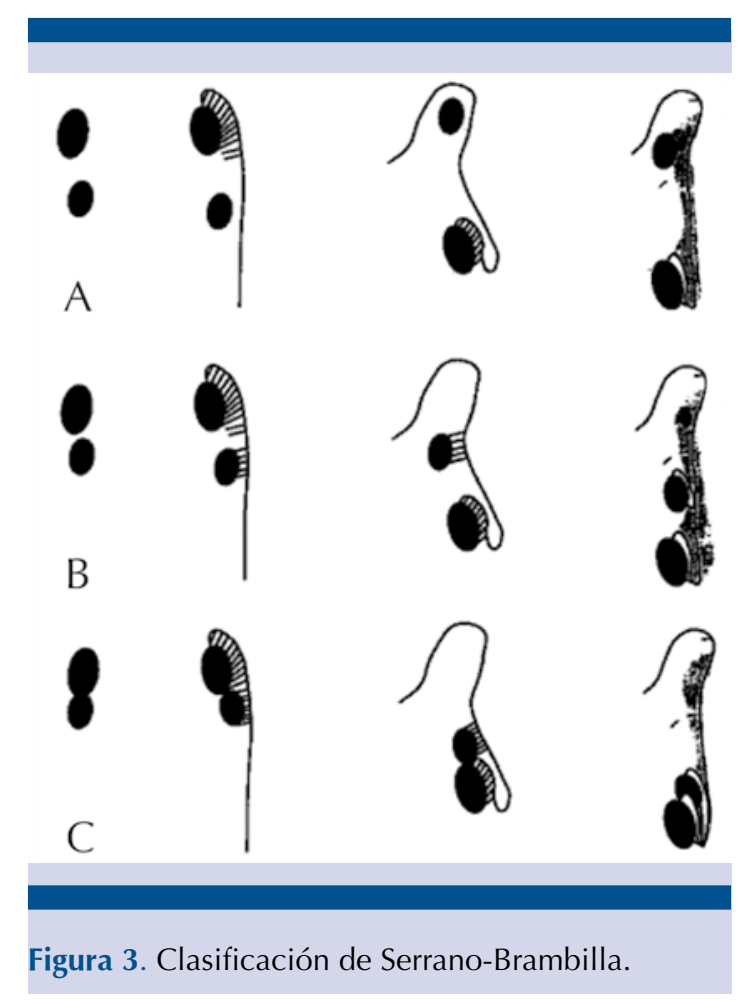


Clasificación de Thum (1991) ${ }^{27}$ con base en el origen embriológico y funcionalidad (Figura 4)

I) El testículo supernumerario no tiene epidídimo ni conducto deferente.

II) El testículo comparte epidídimo y conducto deferente con el testículo ipsilateral.

III) El testículo tiene su propio epidídimo y comparte el conducto deferente.

\section{Clasificación de Singer (1992) ${ }^{28}$ según la}

topografía, anatomía y funcionalidad del testículo supernumerario

I) El testículo tiene conductos de salida para drenar al epidídimo y conducto deferente.

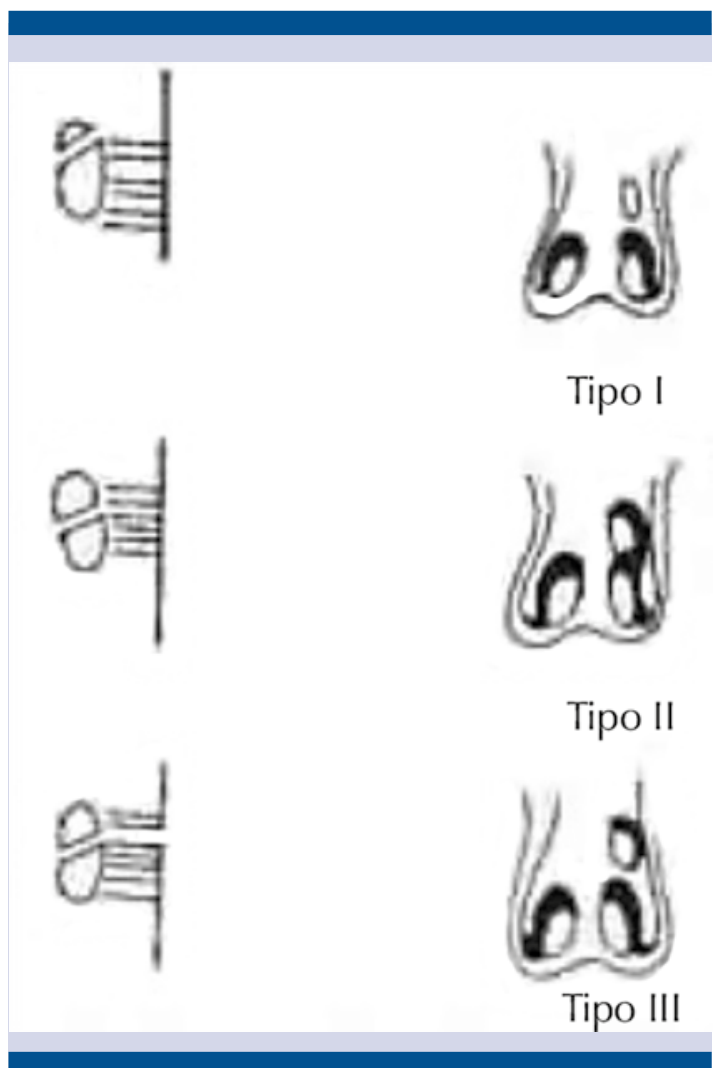

Figura 4. Clasificación de Thum.
IA) Intraescrotal
IB) Inguinal o abdominal (ectópico)

II) El testículo carece de conductos de salida hacia el epidídimo y conducto deferente.

IIA) Intraescrotal

IIB) Ectópico

Clasificación de Bergholz (2007) ${ }^{8}$ con base en su anatomía y función (Figura 5)

A) El testículo drena por el conducto deferente.

A1) Tiene un epidídimo y conducto deferente propio.

A2) Epidídimo propio y conducto deferente compartido con el testículo ipsilateral.

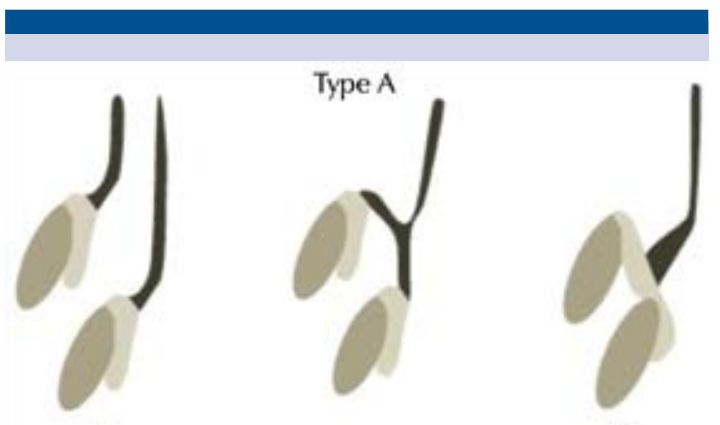

A1

A2

A3
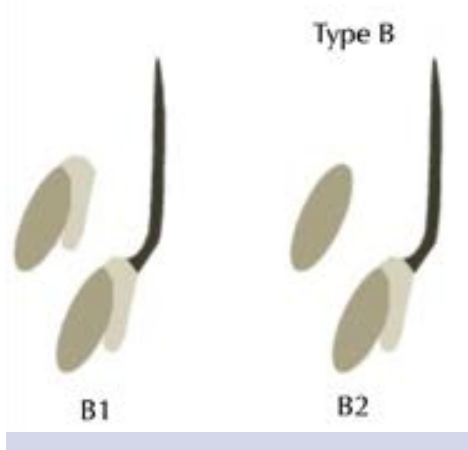

Figura 5. Clasificación de Bergholz. 
A3) Epidídimo y conducto deferente compartido.

B) El testículo no tiene conexión con el conducto deferente.

B1) El testículo supernumerario tiene su propio epidídimo.

B2) El testículo supernumerario no tiene epidídimo, consiste solamente en tejido testicular.

Según la opinión de los autores de este texto, la clasificación de Singer es la más sencilla y de gran utilidad, pues se basa en las dos características fundamentales para la toma de decisiones clínicas, es decir, funcionalidad y localización del testículo supernumerario.

\section{Tratamiento}

Antes del decenio de 1980 el tratamiento inicial consistía en resección quirúrgica; sin embargo, esta modalidad ha cambiado en los últimos años. En pacientes asintomáticos, con testículos supernumerarios de localización escrotal, puede optarse por vigilancia, pues 50\% pueden tener producción de espermatozoides ${ }^{2,8}$ y el riesgo de complicaciones es mínima.

La intervención quirúrgica se realiza en caso de torsión testicular, sospecha de tumoración, testículo localizado en la región inguinal o cuando existe duda acerca de la naturaleza de la masa. Puede obtenerse una biopsia transquirúrgica para comprobar el diagnóstico de testículo supernumerario y descartar la malignidad. En términos generales, se sugiere realizar la resección quirúrgica en caso de un testículo no funcional, independientemente de su localización (Clasificación II de Singer). Cuando el testículo tiene conductos excretores (epidídimo y conducto deferente) y localización ectópica, la mejor opción es realizar orquiectomía (Clasificación IB de Sin- ger), para evitar la evolución a malignidad, casi siempre asociada con tumores seminomatosos. En pacientes con testículos funcionales de localización escrotal, puede realizarse orquidopexia para evitar una futura torsión u orquiectomía en caso de sospecha de malignidad; también debe considerarse el deseo del paciente y de sus padres, pues pueden preferir la permanencia de dos testículos en el escroto, debido a cuestiones estéticas y psicológicas. ${ }^{2}$ Los testículos supernumerarios funcionales, de localización escrotal (Clasificación IA de Singer), no dolorosos, sin sospecha de malignidad, ni problemas psicológicos en el paciente pueden tratarse con vigilancia activa, seguimiento mediante exploración física y estudios ultrasonográficos, como el caso aquí presentado. $^{5}$

Los puntos importantes a recordar en un paciente con testículos supernumerario son:

- La coexistencia de testículo supernumerario debe sospecharse al encontrar una tumoración extratesticular en el área escrotal o inguinal.

- Se recomienda resección quirúrgica de los testículos que se encuentren en la clasificación IB, IIA y IIB de Singer.

- El diagnóstico definitivo se establece mediante estudio histológico del tejido resecado; sin embargo, la sospecha clínica y los hallazgos ultrasonográficos pueden ser suficientes para decidir el tratamiento conservador.

Los autores declaran que no existen conflictos de intereses. No se recibió ningún patrocinio durante la elaboración de este escrito.

\section{REFERENCIAS}

1. Becerra A, Castillo D, Gómez L. Poliorquidismo. Rev Cubana Pediatr. 1998;70:185-188. 
2. Velásquez JG, Flores FR, López LJ. Actas Urol Esp. 2006;30:415-419.

3. Serrano EA, Lorenzo JL, Pérez $\mathrm{HJ}$, et al. Poliorquidismo: informe de un caso y revisión de la bibliografía. Bol Col Mex Urol. 1996;13:141-144.

4. Kumar B, Sharma Ch, Sinha D. Supernumerary testis: a case report and review of literature. J Pediatr Surg. 2008;43:E9-E10.

5. Bergholz R, Wenke K. Polyorchidism: A Meta-Analysis. J Urol. 2009;182:2422-2427.

6. Barrios-Torres E, Eraña-Guerra L, Summano-Avendaño E. Testículos supernumerarios. Bol Med Hosp Infant Mex. 1989;46:56.

7. Janeiro MR, Corominas S, Mallafré JM. Poliorquia: Comunicación de un caso y revisión de la literatura. Bol Col Mex Urol. 1990;7:50.

8. Bergholz R, Koch B, Spieker T. Polyorchidism: a case report and classification. J Pediatr Surg. 2007;42:1933-1935.

9. Mesa D, Pérez M. Poliorquidismo: Reporte de un caso. Acta Pediátrica Costarricense. 1997;11:131-132.

10. Ahmed AH, Elmogy S. P 574- Polyorchidism: A case report in Egypt and review of literature. Fertility and Sterility. 2006;86:S347.

11. Berger $A$, Steiner $H$, Hoeltl $L$, et al. Occurrence of polyorchidism in a young man. Urology. 2002;60:911vi-vii.

12. Lawrentschuk N, MacGregor RJ. Polyorchidism: a case report and review of the literature. Aust N Z J Surg. 2004;74:1130-1132.

13. Orlich C, Murcia O. Poliorquidismo. Acta Med Costarric. 2005;47:197-198.

14. Kumar S, Ranjit P. Polyorchidism: A rare congenital anomaly. Eur J Radiol Extra. 2008;66:e29-e31.

15. García N, Fong F, Santana R, et al. Testículos supernumerarios: anomalía infrecuente del sistema genital. Reporte de caso. Rev Méd Electrón 2011;33:639-643.
16. Arslangou A, Alparslan S, Hamarat M. Polyorchidism: color Doppler ultrasonography and magnetic resonance imaging findings. Clinical Imaging. 2013;37:189-191.

17. Abduljabbar A. A case report: triorchidism; is a rare mistaken cause of extra testicular neoplasm. Urol Case Rep. 2015;3:89-91.

18. Chowdhary S. Case report of a rare variant of polyorchidism. J Ped Surg Case Reports. 2016;5:30-31.

19. Spranger R, Gunst M, Kuhn M. Polyorchidism: A strange anomaly with unsuspected properties. J Urol. 2002;168:198.

20. Repetto P, Ceccarelli P, Bianchini A, et al. Three small testes in left hemiscrotum: a rarer case of polyorchidism. J Pediatr Surg. 2010;45:E21-E23.

21. Alamsahebpour A, Hidas $G$, Kaplan A, et al. Bilateral polyorchidism with diffuse microlithiasis: a case report of an adolescent with 4 testes. J Urol. 2013;82:1421-1423.

22. Prouza A, Stanek Z, Gocalová K, et al. Polyorchidism associated with a solitary testicular myofibroma: Case report and current literature review. Eur Urol Suppl. 2015;14:e1239.

23. Ojili V, Prasad A, Doherty G. An unusual case of polyorchidism with three homolateral testes and contralateral anorchia. Eur J Radiol Extra. 2009;72:e129-e131.

24. Leodoro BM, Beasley SW, Stringer MD. Polyorchidism with presumed contralateral intrauterine testicular torsion. Int J Surg Case Rep. 2014;5:865-867.

25. Woodward PJ, Schwab CM, Sesterhenn IA. Extratesticular Scrotal Masses: Radiologic-Pathologic Correlation 1. Radiographics. 2003;23:215-240.

26. Leung AK. Polyorchidism. Am Fam Physician. 1988;38:153156.

27. Thum G. Polyorchidism: case report and review of literature. J Uol. 1991;145:370-372.

28. Singer BR, Donaldson JG, Jackson DS. Polyorchidism: functional classification and management strategy. Urology. 1992;39:384-388.

\section{AVISO PARA LOS AUTORES}

Revista Mexicana de Urología tiene una nueva plataforma de gestión para envío de artículos. En: https://www.revisionporpares.com/index.php/RMUrol podrá inscribirse en nuestra base de datos administrada por el sistema Open Journal System (OJS) que ofrece las siguientes ventajas para los autores:

- Subir sus artículos directamente al sistema.

- Conocer, en cualquier momento, el estado de los artículos enviados, es decir, si ya fueron asignados a un revisor, aceptados con o sin cambios, o rechazados.

- Participar en el proceso editorial corrigiendo y modificando sus artículos hasta su aceptación final. 